\title{
O livro do conhecimento: um jogo de aventura para exercitar a ortografia
}

\author{
Fernanda Gabriela S. Pires ${ }^{1,2,3}$, Fábio Michel $^{1,2}$, João Ricardo Serique Bernardo ${ }^{1,2}$, \\ Rafaela Melo Ferreira ${ }^{1,2}$ Rosiane de Freitas $^{3}$ \\ ${ }^{1}$ Licenciatura em Computação - Universidade do Estado do Amazonas (UEA) \\ Av. Darcy Vargas, 1.200- 69050-020 - Manaus - AM - Brazil \\ ${ }^{2}$ Laboratório de Tecnologias Educacionais - Universidade do Estado do Amazonas \\ Manaus, AM - Brazil \\ ${ }^{3}$ Instituto de Computação - Universidade Federal do Amazonas (UFAM) - Manaus, AM \\ - Brazil \\ \{fpires, fmmdl.lic16, jrsbr.lic16, rmf.lic16\} duea.edu.br, \\ rosianedicomp. ufam.edu.br.
}

\begin{abstract}
This article reports on the process of development of the educational purpose game "The Knowledge Book", whose objective is to exercise the spelling in Portuguese in a ludified way, motivating the players to overcome the proposed challenges. It is an electronic platform game, action-adventure style, whose purpose is to assist the user in the process of spelling learning. The software was developed using the game engine tool Construct 2 and followed the agile development model. It is based on the Cognitive Theory of Learning and Multimedia (TCAM) and Active Learning, in what concerns the development of the interface the indicated method for application. Preliminary tests point out that the elaborated game can generate positive impacts and enhance learning.
\end{abstract}

Resumo. Este artigo relata o processo de desenvolvimento do jogo de propósito educacional "O Livro do Conhecimento", com a finalidade de exercitar a ortografia em língua portuguesa de forma ludificada, motivando os jogadores a vencer os desafios propostos. É um jogo eletrônico de plataforma, estilo açãoaventura, que pretende auxiliar o usuário no processo de aprendizagem em ortografia. O software foi desenvolvido por meio da ferramenta game engine Construct 2 e seguiu o modelo de desenvolvimento ágil. Está fundamentado na Teoria Cognitiva de Aprendizagem e Multimídia(TCAM) e processo de aprendizagem ativa, no que tange o desenvolvimento da interface o método indicado para aplicação. Testes preliminares apontam que o jogo elaborado pode gerar impactos positivos e potencializar a aprendizagem.

\section{Introdução}

A Avaliação Nacional de Alfabetização (ANA), revelou que 34,46\% dos brasileiros não possuem rendimento adequado na escrita INEP (2016), em 2016, o rendimento na prova de redação do Exame Nacional do Ensino Médio (ENEM) foi um dos piores nos últimos 3 anos. Os números são preocupantes, pois revelam que a base nacional da educação passa por graves dificuldades. Considerando tais índices, diversos recursos didáticos têm sido produzidos com a finalidade de auxiliar no aprendizado de língua portuguesa.

$\mathrm{Na}$ era da tecnologia digital, as pessoas tem passado muito tempo jogando, por 
VII Congresso Brasileiro de Informática na Educação (CBIE 2018)

Anais do XXIX Simpósio Brasileiro de Informática na Educação (SBIE 2018)

que não aplicar o mesmo empenho a outras tarefas, como os estudos (McGonigal, 2017). Pesquisas vem sendo realizadas com a finalidade de unir aprendizagem a diversão, por meio de jogos eletrônicos. O objetivo é que o mesmo engajamento empregado nos jogos para entretenimento possa ser alcançado naqueles cujo objetivo é a aprendizagem, os intitulados jogos com propósito sério do inglês Serious Game (Djaouti, Alvarez, Jessel, \& Rampnoux, 2011).

Mediante o cenário descrito a cima, o presente artigo trata da concepção e desenvolvimento de um jogo de plataforma no estilo ação-aventura com propósito educacional, intitulado "O Livro do Conhecimento", que tem como finalidade auxiliar na aprendizagem de Língua Portuguesa, com foco nas habilidades ortográficas. Seu desenvolvimento levou em consideração os requisitos cognitivos de aprendizagem, os fatores de Interação Humano Computador (IHC) e elementos de gamificação. A mecânica do jogo proposto apresenta elementos de exploração, aventura, progressão do herói e combates, levando o jogador a um processo de imersão, em que se espera, sejam consolidadas as memórias de longo prazo, para que ocorra a aprendizagem.

O restante deste artigo está organizado da seguinte forma: na Seção 2 são apresentados os trabalhos relacionados; na Seção 3 é dada a fundamentação teórica necessária para o desenvolvimento do jogo, com a Teoria da Aprendizagem Multimídia de Richard Mayer (2009). Na Seção 4, o projeto do jogo é descrito, tratando-se do enredo do jogo, cenários das fases, conteúdos de aprendizagem e mecânica geral envolvida. Na seção 5 são apresentados os resultados bem como avaliações de usabilidade e na Seção 6 são apresentadas as considerações finais .

\section{Trabalhos relacionados}

Levando em consideração as dificuldades ortográficas existentes, Assis et al. (2017) propuseram a utilização de um aplicativo para dispositivos móveis intitulado "Grapphia", voltado para o conteúdo de ortografia de Língua Portuguesa. O foco do aplicativo é a escrita de palavras com dígrafos ou letras que possuem o mesmo som e seu público alvo são crianças de 8 a 10 anos.

O crescente incentivo a utilização de dispositivos móveis no cenário educacional, resultou na proposta do serious game "AmarganA" por Yamato, Corrêa, \& Martins (2017). O jogo foi desenvolvido para plataformas iOS e tem por objetivo auxiliar no processo de aprendizagem de ortografia, de maneira a torná-lo imersivo e interativo. A partir dos testes aplicados com crianças no ensino fundamental, os autores chegaram a conclusão de que o aplicativo é capaz de proporcionar a aprendizagem de Língua Portuguesa e incentivar a criatividade.

Oliveira, Castro, Ferreira, \& Ouverney-King (2017) desenvolveram o aplicativo mobile "Super-hífen" com a finalidade de auxiliar na aprendizagem da nova ortografia da Língua Portuguesa, com foco na utilização do hífen. O jogo foi construído na ferramenta Construct 2 e procura promover reflexões acerca do conteúdo de forma lúdica e motivacional. A partir de testes realizados, os autores obtiveram resultados positivos quanto a usabilidade e interatividade. A ferramenta é um recurso alternativo de aprendizagem e aproveita os elementos presentes em jogos digitais para o processo de construção do conhecimento, tornando-o divertido. 
VII Congresso Brasileiro de Informática na Educação (CBIE 2018)

Anais do XXIX Simpósio Brasileiro de Informática na Educação (SBIE 2018)

\section{Jogos eletrônicos e processos cognitivos de aprendizagem}

\subsection{Jogos eletrônicos}

Huizinga (2014) destaca os jogos como uma atividade voluntária do ser humano, por se tratar de algo lúdico, divertido e cômico, sua existência é anterior a cultura, segundo o mesmo autor. A origem dos jogos eletrônicos remonta os anos 1950, quando os computadores ainda habitavam somente os grandes centros de pesquisa e as telas de exibição era osciloscópios (Rogers, 2014).

Com o passar do tempo e a evolução das tecnologias eles puderam chegar a casa das pessoas através dos consoles, árcades e jogos de computador. Os jogos eletrônicos digitais são todos aqueles que pode ser acompanhado via tela de vídeo. Eles têm alcançado um número cada vez maior de pessoas que dispendem um grande número de horas engajadas em missões virtuais, em outros "mundos", criando relações interpessoais entre os seu avatares, salvando pessoas, vencendo guerras, etc (McGonigal, 2017).

\subsection{Serious Game e Aprendizagem}

Jogos sérios do inglês serious game, segundo Abt (1987) são aqueles cujo objetivo principal não é o entretenimento e sim a aprendizagem de forma lúdica, cujo planejamento repousa sobre os objetivos de aprendizagem, mais recentemente o termo também se refere aos jogos digitais voltados a aprendizagem neste mesmo âmbito (Djaouti et al., 2011).

O Livro do Conhecimento, aqui defendido é um jogo de propósito sério, que tem como objetivo auxiliar no processo de aprendizagem em Língua Portuguesa, desenvolvido para computador. É classificado como um jogo de plataforma aventuraação, definido por Rogers (2014) como aqueles que envolvem quebra cabeças e objetivos baseados em histórias cujo cenário é formado por plataformas em que o personagem principal anda, pula, ou quica em busca de seu objetivo.

Muitos estudiosos têm discutido os jogos como ferramenta auxiliar no processo de aprendizagem (Kafai \& Burke, 2015; Subrahmanyam \& Renukarya, 2015). Os jogos despertam o interesse dos alunos pelo fato de se mostrarem mais atrativos do que os elementos presentes no ambiente escolar. Ribeiro \& Galera (2009) explicam que, devido a isso, os jogos se apresentam como uma alternativa de auxílio para a aprendizagem, desenvolvendo habilidades de lógica e resolução de problemas, em diversas áreas do conhecimento.

\subsection{Língua Portuguesa e aprendizagem}

Aprender a comunicar-se através da fala e da escrita, é uma habilidade construída através de uma rede simbólica de significados cuja configuração existe no sistema central de regras de um idioma (Carraher, 2012). Lorensatti (2009) reforça que a aprendizagem da Língua Portuguesa é essencial no processo de comunicação oral ou escrito, além do quê, a língua é o que define uma nação.

No Exame Nacional do Ensino Médio (ENEM) aplicado no ano de 2017, dos 4.714.558 milhões de participantes, apenas 53 obtiveram a nota máxima na prova de redação, o que representa $0,001 \%$. Além disso, 309.157, cerca de 6,5\% dos participantes, zeraram a prova de redação. Em 2018, dados do Índice Nacional de Analfabetismo Funcional (INAF) revelaram que a taxa de analfabetismo funcional (dificuldade de leitura e escrita em atividades cotidianas) dentre os brasileiros é de $29 \%$ e que $8 \%$ da população 
VII Congresso Brasileiro de Informática na Educação (CBIE 2018)

Anais do XXIX Simpósio Brasileiro de Informática na Educação (SBIE 2018)

brasileira é classificada como analfabeta (não sabem ler e escrever).Os recentes dados negativos mobilizaram vários pesquisadores a discutir como desenvolver metodologias que possam auxiliar na aprendizagem da língua portuguesa. (Lorensatti, 2009; Oliveira et al., 2017; Yamato et al., 2017).

\subsection{Cognição e Processo de aprendizagem}

Ao avaliar a forma como as pessoas aprendem, Mayer (2009) defende que a aprendizagem ocorre de forma eficiente quando a apresentações de conteúdo é realizada por meio de palavras e imagens . A Teoria Cognitiva da Aprendizagem Multimídia (TCAM) (Mayer, 2009) explica que estímulos nas memórias, sensorial e de trabalho, são fundamentais para os processos que possibilitam ao usuário desenvolver memórias de longo prazo. Isso acontece através de associações, realizadas entre palavras e imagens, processadas pelo sistema cognitivo humano.

Considerando as bases biológicas da aprendizagem e leitura, defendidas pela neurociência, faz-se necessário o uso de interfaces gráficas capazes de prover compreensão cabível ao córtex occípito-temporal esquerdo, pois é sobre o córtex que repousa a aprendizagem, por ser a região com o maior número de neurônios especializados. São eles que possuem o maior contato com as entradas visuais (Dehaene, 2012).

A teoria cognitiva da aprendizagem multimídia destaca que, ao desenvolver materiais multimídia, devem ser levados em conta 12 princípios pois os usuários sofrem 3 tipos de processamento cognitivo durante a aprendizagem: Redução do Processamento Estranho, Gerenciamento do Processamento Essencial e Processamento Generativo (Sweller, 2011). Na redução do processamento estranho, Mayer (2009) explica que informações desnecessárias devem ser excluídas do processo de aprendizagem. No processamento cognitivo essencial as informações prévias e necessárias devem ser repassadas de maneira adequada para cada aluno e no processamento Generativo isso deve ser considerado, por ser inerente as estruturas adquiridas.

\section{O jogo de ação-aventura proposto: "O Livro do Conhecimento"}

Aqui é apesentado o jogo de propósito educacional sério "O Livro do Conhecimento" Sua mecânica é baseada em sucessos mundialmente conhecidos como Super Mario World e CupHead. Nesses jogos, a característica marcante é proporcionar uma experiência de jogo simples e divertida, além de possuir uma história motivadora que que incentiva a imersão no universo apresentado, é indicado para usuários a partir de 10 anos de idade. $\mathrm{O}$ jogo está dividido em três fases, na primeira Diana enfrenta os Trolls, na segunda a batalha é com os Orcs, e na terceira fase ela enfrenta o chefão Valtor.

No software $O$ Livro do Conhecimento são aplicados os pressupostos da Teoria da Carga Cognitiva (Sweller, 2011) e os pressupostos da Teoria cognitiva da aprendizagem multimídia (Mayer, 2009), através de uma proposta de interface imersiva, sem a sobrecarga de informação por unidade de tempo(chunk), com a finalidade de potencializar a Aprendizagem.

Para que $O$ Livro do Conhecimento se tornasse atrativo e divertido, sem perder o foco da aprendizagem, realizou-se uma análise do jogo, contendo informações para detalhar o enquadramento, conforme os conceitos determinados como fundamentais para 
VII Congresso Brasileiro de Informática na Educação (CBIE 2018)

Anais do XXIX Simpósio Brasileiro de Informática na Educação (SBIE 2018)

um jogo ou plataforma Gamificada (Alves, 2015).

A tabela 1, apresenta a organização dos personagens, elementos que configuram a mecânica, cenário, e coletáveis bem como sua função no cenário do jogo. Observa-se, através da representação visual que todos os elementos do jogo bem como a game play apresentada, foi construída tendo como base o storytelling desenvolvido, na era medieval.

Tabela 1. Elementos presents no jogo e suas funções

\begin{tabular}{|c|c|}
\hline Assets & Caracteristicas \\
\hline $\begin{array}{l}\text { Princesa Diana } \\
\text { (Personagem principal) }\end{array}$ & $\begin{array}{l}\text { A personagem principal tem características humanas com estilo medieval, } \\
\text { a mesma possui um arco e flecha que é controlado pelo jogador. }\end{array}$ \\
\hline \multirow[t]{4}{*}{ Inimigos } & Valtor (Elfo) \\
\hline & Trolls ( $1^{\mathrm{a}}$ Fase) \\
\hline & Ores ( $2^{\mathrm{a}}$ Fase $)$ \\
\hline & Trolls, Ores e Elfos ( $3^{a}$ Fase $)$ \\
\hline \multirow[t]{2}{*}{ Cenários } & Primeira fase: Floresta aos arredores do reino. \\
\hline & Segunda e Terceira fase: Muro de entrada do reino de Damaris \\
\hline \multirow[t]{3}{*}{ Objetos de cenário } & Fase 1: Arvores, lagos, abismos, pedras, blocos e plataformas montáveis. \\
\hline & Fase 2: abismos, blocos e plataformas montáveis. \\
\hline & Fase 3: plataforma que se movimenta em posição vertical. \\
\hline \multirow[t]{4}{*}{ H.U.D. (Head Up Displa } & $\begin{array}{l}\text { Barra de vida (demonstra o nível de vida, em questão de ataques de } \\
\text { inimigos). }\end{array}$ \\
\hline & Numero de vidas: (indica o número de vidas que o player ainda possui). \\
\hline & $\begin{array}{l}\text { Papiros recolhidos: (demonstra a quantidade de papiros que o player } \\
\text { adquiriu pela fase) }\end{array}$ \\
\hline & Barra de moedas: (indica o número de joias que o player coletou) \\
\hline \multirow[t]{3}{*}{ Itens coletaveis } & Joias e saco de jóias (coins) \\
\hline & Papiro (Página do livro do conhecimento) \\
\hline & Maças (Recuperação de energia) \\
\hline
\end{tabular}

\subsubsection{A implementação do jogo}

Nesta seção, são apresentadas as decisões de projeto e os detalhes de implementação do jogo proposto. Após a prototipação e definição do estilo de jogo, plataforma $2 \mathrm{D}$, iniciouse a fase de desenvolvimento. A primeira etapa consistiu na montagem dos personagens e cenários, usou-se a técnica de composição através de imagens e sprites de livre acesso na web. O segundo momento consistiu na escolha do ambiente de desenvolvimento, a game engine, selecionada foi a Construct $2^{1}$, considerando desempenho, qualidade e facilidade do processo de produção com um sistema de programação visual através de eventos e ações definidas pelos desenvolvedores que atende a ao desenvolvimento de jogos 2D, possibilitando exportar para diversas plataformas como smartphones Android e iOS, sistemas Windows, Mac, Linux e interfaces Web. Contudo, a primeira versão do aplicativo é limitada para computadores, com uma versão mobile em processo de desenvolvimento.

\footnotetext{
${ }^{1}$ Disponível em: https://www.scirra.com/construct2
} 
VII Congresso Brasileiro de Informática na Educação (CBIE 2018)

Anais do XXIX Simpósio Brasileiro de Informática na Educação (SBIE 2018)

Foram ainda utilizadas outras ferramentas durante o processo de desenvolvimento, conforme demonstrado na tabela 2.

Tabela 2. Ferramentas utilizadas na construção do jogo.

\begin{tabular}{lll}
\hline \multicolumn{1}{c}{ Ferramenta } & \multicolumn{1}{c}{ Etapa } & \multicolumn{1}{c}{ Função } \\
\hline Trello & Organização do game & Gerência da construção do projeto \\
PowerPoint 2016 & Mockup & Demo do gameplay, mecânica \\
Adobe Photoshop CC 2017 & Edição de imagens & Edição gráfica de personagens, cenários, \\
Adobe Illustrator CC 2017 & Edição de imagens & botões e assets. \\
Construct & Construção de jogos & Desenvolvimento do jogo \\
\hline
\end{tabular}

\subsubsection{Processo de teste de software e validação}

Para que os jogadores tenham uma experiência de jogo adequada, a usabilidade é essencial, se mal elaborada pode causar desvio de atenção (Gurgel, Arcoverde, Almeida, Sultanum, \& Tedesco, 2006). Com o objetivo de avaliar o nível de diversão, imersão e prazer que o jogo de aprendizagem proporciona, foi utilizado o método E-Game Flow (Sweetser \& Wyeth, 2005), que objetiva aplicar heurísticas quanto à usabilidade e experiência do jogador. O método E-Game Flow tem como base a Teoria do Fluxo e possui a finalidade de explicar características que tornam agradável determinada atividade. O psicólogo Mihaly Csikszentmihalyi (Sweetser \& Wyeth, 2005), destacou o estado de Fluxo como uma experiência gratificante em que o usuário se sente imerso na realização de uma atividade por vontade própria.

Para a avaliação de jogos, o método E-Game Flow propõe 8 categorias: Concentração, Desafio, Habilidades do Jogador, Controle, Objetivos, Feedback, Imersão e Interação (Sweetser \& Wyeth, 2005). A Tabela 3 a seguir descreve cada critério:

Tabela 3. Tabela de Critérios do E-Game Flow.

\begin{tabular}{ll}
\hline \multicolumn{1}{c}{ Critério } & \multicolumn{1}{c}{ Descrição } \\
\hline $\begin{array}{l}\text { Concentração } \\
\text { Desafio }\end{array}$ & $\begin{array}{l}\text { Retenção do foco e atenção do usuário, sem sobrecarga com tarefas não essenciais. } \\
\text { Os desafios não devem oferecer uma pressão excessiva e nem gerar apatia. } \\
\text { Habilidades do } \\
\text { jogador }\end{array}$ \\
$\begin{array}{l}\text { A experiência de jogo deve fornecer tarefas que proporcionem o desenvolvimento de } \\
\text { habilidades do jogador, através de um processo divertido e motivador. }\end{array}$ \\
Objetivos & $\begin{array}{l}\text { O jogador deve possuir controle sobre os elementos e objetivos do jogo. } \\
\text { Odequados. }\end{array}$ \\
Feedback & $\begin{array}{l}\text { São as informações que o jogador deve receber acerca do seu progresso e resultados } \\
\text { das ações de suas tarefas. }\end{array}$ \\
Imersão & $\begin{array}{l}\text { Os jogadores se sentem transportados ao universo do game, fazendo com que o usuário } \\
\text { perca o senso de tempo e consciência do que acontece à sua volta. }\end{array}$ \\
Interação & O jogo proporciona oportunidades de interação com outros jogadores, competição, \\
salas de chat online e etc.
\end{tabular}

\subsection{Enredo}

A história se passa na era medieval, em um reino chamado Damaris que tem a posse do Livro do Conhecimento. O poder do artefato, permite aos súditos falar e escrever em Língua Portuguesa corretamente. No entanto, seres malignos desejam a posse do livro. Valtor, o principal vilão, reúne uma tropa de Elfos, Trolls e Orcs que avançam sobre 
Damaris para tomar o Livro.

Durante a batalha, o rei é capturado, a rainha e suas duas filhas, Miriam e Diana fogem para a floresta. No entanto, são capturadas logo em seguida e apenas a princesa, Diana, consegue fugir, sendo encontrada por um sábio por quem é treinada. Valtor é bemsucedido em sua investida e domina o reino, furta "O Livro do Conhecimento" que passa a ser utilizado para o mal e todos começam a se expressar de maneira incorreta. A princesa passou anos treinando arduamente, com o objetivo de combater seus inimigos, enfrentar diversos desafios, recuperar o livro e retomar o controle do reino onde cresceu. Neste jogo, o jogador vive o papel da princesa Diana, em sua jornada para trazer a paz ao seu reino, recuperar o livro permitindo que as pessoas falem corretamente como outrora. Ao percorrer a plataforma, a heroína captura fragmentos do livro que ajudarão no combate final com o vilão, pelos conhecimentos presentes em suas páginas, são como bônus que aumentam as possibilidades de vitória.

\subsection{Descrição do jogo}

Os princípios da teoria da carga cognitiva e aprendizagem multimídia estão presentes em todas as fases do game, pois quando se faz necessário completar um obstáculo ou liberar uma parte do trajeto, ocorre aprendizagem por meio do uso de palavras, sons e imagens, além do envolvimento emocional que o jogador é levado a ter, através do enlace de pensamentos e lembranças, frutos da experiência vivida ao longo do jogo.

Durante o percurso há desafios nas fases que estimulam o jogador a superá-los, usando seu conhecimento sobre ortografia. Por exemplo, ao se deparar com uma plataforma que está desabilitada, ou bloqueando alguma passagem, o obstáculo irá conter uma palavra com uma interrogação. Para alcançar seu objetivo, o jogador deve escolher a letra correta. Em outros obstáculos, o jogador encontrará palavras que estão escritas de maneira correta e outras de forma incorreta, para avançar o usuário deve usar apenas as que estão escritas corretamente. A cada obstáculo superado o jogador se aproxima das páginas perdidas do livro e ao encontrar uma página, obtém diferentes informações, que mostram a distinção entre palavras que possuem escrita parecida, como por exemplo: "a gente" e "agente"; "mas" e "mais"; "esse" e "este", etc.

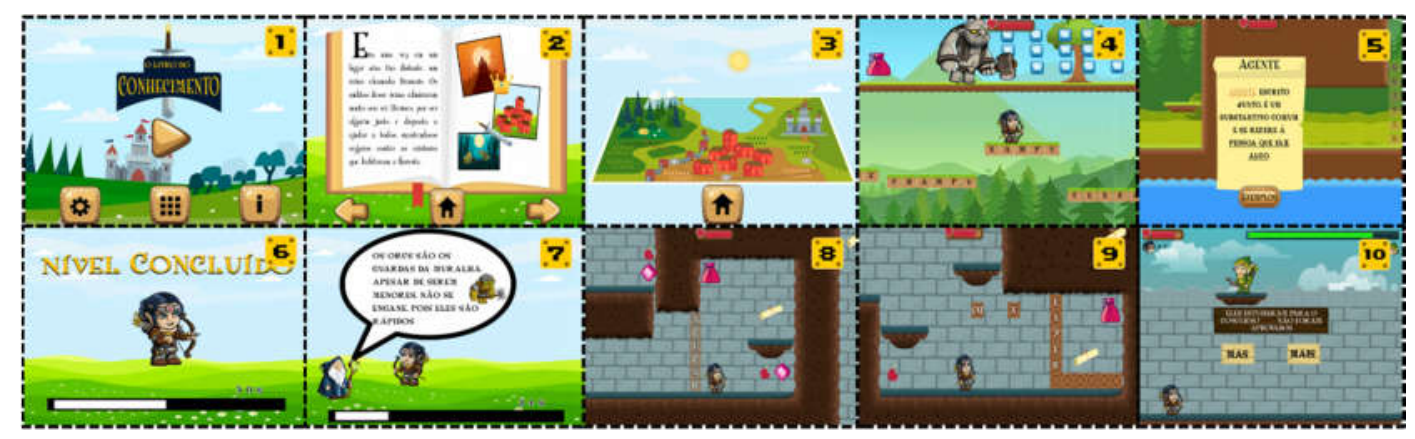

Figura 1. Jornada do jogador (Sequência de fases 1-3)

O jogador possui um "arco e flecha" que pode usar contra seus inimigos. Para derrotar cada monstro é preciso atirar flechas. Há também jóias que podem ser coletadas e, a cada 100 adquiridas, o jogador ganha uma vida. As maçãs coletadas recuperam a energia da guerreira. À medida que combate avança, surgem perguntas referentes aos conhecimentos adquiridos ao longo das fases dispostos nas páginas recuperadas do livro. 
VII Congresso Brasileiro de Informática na Educação (CBIE 2018)

Anais do XXIX Simpósio Brasileiro de Informática na Educação (SBIE 2018)

Para responder à pergunta, o jogador deve pular e bater na caixa referente à resposta que escolher. Se errar, a punição que receberá é o restabelecimento da energia do chefão.

\section{Resultados e Avaliações}

\subsection{Enquadramento Teoria Cognitiva da Aprendizagem Multimídia}

Durante o processo de desenvolvimento e prototipação, foram aplicados testes com a finalidade de garantir que a disposição da interface estivesse de acordo com a TCAM, para garantir adesão ao modelo de processamento da informação em dispositivos multimídia. A Tabela 1 mostra o enquadramento do jogo O Livro do Conhecimento, em cada um dos 12 princípios de Richard Mayer, atingindo um total de 91,6\% de metas propostas:

Tabela 4. Enquadramento dos princípios do processamento da informação de Richard Mayer.

\begin{tabular}{llc}
\hline \multicolumn{2}{c}{ Princípios } & Status \\
\hline & Coerência & 1 \\
Princípios para reduzir o & Sinalização & 1 \\
processamento estranho & Redundância & 1 \\
& Contiguidade Espacial & 1 \\
& Contiguidade Temporal & 1 \\
\hline Princípios para & Segmentação & 1 \\
Gerenciamento do & Pré-Formação & 1 \\
Processamento Essencial & Modalidade & 1 \\
\hline \multirow{3}{*}{ Princípios para Promover o } & Multimídia & 1 \\
Processamento Generativo & Vozsonalização & 1 \\
& Imagem & 0 \\
\hline Legenda: 1 = Possui; 0 = Não Possui.
\end{tabular}

\subsection{Avaliação de Usabilidade}

Com base nas categorias propostas pelo método E-Game Flow (Sweetser \& Wyeth, 2005), a Tabela 3, apresentada a seguir, demonstra a pontuação alcançada pelo Livro do Conhecimento, em cada critério, após a avaliação de 12 jogadores que participaram da experiência de utilizá-lo:

Tabela 5. Tabela de avaliação E-Game Flow apresenta resultados de avaliação pelos usuários.

\begin{tabular}{lccccc}
\hline \multirow{2}{*}{ Critério } & \multicolumn{5}{c}{ Porcentagem de notas dos avaliadores } \\
\cline { 2 - 6 } & Não, se aplica & Deveria ter, mas não tem & Ruim & Bom & Ótimo \\
\hline Concentração & $2 \%$ & $12 \%$ & $8 \%$ & $46 \%$ & $32 \%$ \\
Desafio & $13 \%$ & $14 \%$ & $10 \%$ & $23 \%$ & $40 \%$ \\
Habilidades do jogador & $14 \%$ & $11 \%$ & $15 \%$ & $24 \%$ & $36 \%$ \\
Controle & $7 \%$ & $10 \%$ & $21 \%$ & $33 \%$ & $29 \%$ \\
Objetivos & $0 \%$ & $4 \%$ & $8 \%$ & $58 \%$ & $29 \%$ \\
Feedback & $8 \%$ & $3 \%$ & $17 \%$ & $33 \%$ & $39 \%$ \\
Imersão & $19 \%$ & $4 \%$ & $17 \%$ & $40 \%$ & $20 \%$ \\
Interação & $42 \%$ & $30 \%$ & $28 \%$ & $0 \%$ & $0 \%$ \\
\hline
\end{tabular}


VII Congresso Brasileiro de Informática na Educação (CBIE 2018)

Anais do XXIX Simpósio Brasileiro de Informática na Educação (SBIE 2018)

As notas revelam que o jogo "O Livro do Conhecimento" atingiu as seguintes porcentagens, no intervalo entre bom e ótimo, nos seguintes critérios: $78 \%$ concentração, $63 \%$ desafios apresentados, $60 \%$ em Habilidades do jogador, $62 \%$ em Controle, $87 \%$ nos objetivos, 72 de feedback, $60 \%$ de imersão e $0 \%$ em interação.

As categorias que tiveram destaque positivo foram a concentração, objetivos e feedback. Desafios, habilidades do jogador, controle e imersão, indicam que ajustes são necessários. A interação recebeu $0 \%$, dentro do intervalo destacado, pois o jogo não oferece um modo multiplayer online, chat ou salas de bate papo.

Os resultados obtidos foram necessários para ajustes e melhorias no processo de desenvolvimento. Entretanto, os usuários mostraram-se satisfeitos com o uso do jogo, história envolvida e simplicidade na mecânica apresentada. Porém, foi detectada a necessidade de adição de novas fases e uma versão para dispositivos móveis.

O jogo foi avaliado segundo a tabela de gamificação definida por Flora Alves (Alves, 2015), que delimita os requisitos esperados de um jogo. Na categoria "mecânica", atingiu-se 55,5\% dos requisitos postulados: desafios disponíveis, feedback, recompensas do jogo e estados de vitória. Na categoria "dinâmica" o jogo atingiu 100\% dos requisitos: progressão do jogo, emoções e narrativa. Para trabalhar com os componentes do objeto de aprendizagem, foi alcançado mais de $71,4 \%$ de elementos como: combate, pontuação e avatares. Todos os elementos avaliados na tabela de enquadramento, baseada em (Alves, 2015), serviram de auxílio no levantamento de características do software, com relação as mecânicas de jogos desejáveis e atingidas.

\section{Considerações Finais}

Este artigo teve como finalidade apresentar a concepção, desenvolvimento e análise de usabilidade do jogo "O livro do Conhecimento". Desenvolvido para proporcionar uma experiência de aprendizagem, divertida e gamificada, tendo como referências jogos mundialmente conhecidos. $\mathrm{O}$ jogo pode ser utilizado como ferramenta de auxílio à aprendizagem em Língua Portuguesa. O jogador é motivado a vencer os desafios, gradativamente, em cada etapa, dentro do processo de aprendizagem proporcionado pelo ambiente.

Ressalta-se ainda o modelo de desenvolvimento próprio, a partir do qual o jogo foi construído, aliando processo ágil de desenvolvimento de software, teorias de aprendizagem a serem consideradas tendo como base estudos em neurociência, processo de teste de software com vistas ao desenvolvimento de um produto que atendesse as necessidades de aprendizagem de forma lúdica, a proposta é que a aprendizagem ocorra de forma implícita e não explicita, promovendo com isso uma imersão prazerosa aumentando as possibilidades de se atingir o engajamento durante a execução das atividades. As avalições de usabilidade dos usuários apontaram a necessidade de poucos ajustes, mas indicaram principalmente o sucesso em alcançar uma mecânica gamificada sem perder o propósito da aprendizagem.

Vale ressaltar que o objetivo do desenvolvimento do software é fornecer uma ferramenta de auxílio a aprendizagem, que auxilie na escrita correta da língua portuguesa, estrutura de palavras e aplicações em frases cotidianas.

\section{Referências}


VII Congresso Brasileiro de Informática na Educação (CBIE 2018)

Anais do XXIX Simpósio Brasileiro de Informática na Educação (SBIE 2018)

Abt, C. C. (1987). Serious Games: University Press of America.

Alves, F. (2015). Gamification: Como criar experiencias de aprendizagem engajadoras: DVS Editora.

Assis, L., Bodolay, A., Gregório, L., Santos, M., Vivas, A., Pitangui, C., \& Bandeira, D. (2017). Grapphia: Aplicativo para Dispositivos Móveis para Auxiliar o Ensino da Ortografia. Paper presented at the Anais dos Workshops do Congresso Brasileiro de Informática na Educação.

Carraher, T. N. (2012). Explorações sobre o desenvolvimento da competência em ortografia em português. Psicologia: teoria e pesquisa, 1(3), 269-285.

Dehaene, S. (2012). Os neurônios da leitura. Porto Alegre: Penso.

Djaouti, D., Alvarez, J., Jessel, J.-P., \& Rampnoux, O. (2011). Origins of serious games Serious games and edutainment applications (pp. 25-43): Springer.

Gurgel, I., Arcoverde, R. L., Almeida, E. W., Sultanum, N. B., \& Tedesco, P. (2006). A importância de avaliar a usabilidade dos jogos: a experiência do Virtual Team. Anais do SBGames, Recife.

Huizinga, J. (2014). Homo Ludens: o jôgo como elemento da cultura. Brasil: Perspectiva.

INEP. (2016). Resultados da ANA 2016 por estados e municípios estão disponíveis no Painel Educacional do Inep. from http://portal.inep.gov.br/artigo//asset publisher/B4AQV9zFY7Bv/content/resultados-da-ana-2016-por-estadose-municipios-estao-disponiveis-no-painel-educacional-do-inep/21206

Kafai, Y. B., \& Burke, Q. (2015). Constructionist gaming: Understanding the benefits of making games for learning. Educational psychologist, 50(4), 313-334.

Lorensatti, E. J. C. (2009). Linguagem matemática e Língua Portuguesa: diálogo necessário na resolução de problemas matemáticos. CONJECTURA: filosofia e educação, 14(2).

Mayer, R. E. (2009). Multimedia learning: Cambridge university press.

McGonigal, J. (2017). A realidade em jogo: Best Seller.

Oliveira, A. C. C., Castro, M. d. G. A., Ferreira, L. D. S., \& Ouverney-King, J. (2017). Super-hifen: gamifying the hyphen. Paper presented at the Brazilian Symposium on Computers in Education (Simpósio Brasileiro de Informática na EducaçãoSBIE).

Ribeiro, R. J., \& Galera, J. M. B. (2009). Jogos epistêmicos para educação.

Rogers, S. (2014). Level Up! The Guide to Great Video Game Design: Wiley.

Subrahmanyam, K., \& Renukarya, B. (2015). Digital games and learning: Identifying pathways of influence. Educational psychologist, 50(4), 335-348.

Sweetser, P., \& Wyeth, P. (2005). GameFlow: a model for evaluating player enjoyment in games. Computers in Entertainment (CIE), 3(3), 3-3.

Sweller, J. (2011). Cognitive load theory Psychology of learning and motivation (Vol. 55, pp. 37-76): Elsevier.

Yamato, E. M., Corrêa, A. G. D., \& Martins, V. F. (2017). AmarganA: A spelling game of the Portuguese language for use in mobile devices. Paper presented at the Information Systems and Technologies (CISTI), 2017 12th Iberian Conference on. 